\title{
The initial diagnosis and management of Parkinson's disease
}

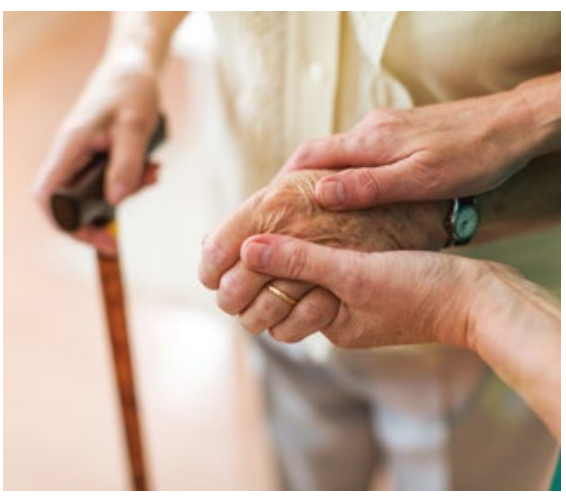

CPD

Sophie Waller, Laura Williams,

Hugo Morales-Briceño, Victor SC Fung

\section{Background}

Parkinson's disease is a common, progressive neurodegenerative disorder, the prevalence of which is on the rise. The diagnosis and management of Parkinson's disease is therefore likely to become increasingly frequent in general practice.

\section{Objective}

The aim of this article is to provide a practical overview for the general practitioner of the initial diagnosis and management of Parkinson's disease.

\section{Discussion}

Parkinson's disease is a multisystem disorder, and the way the diagnosis is delivered, as well as the early management, can have a lasting impact on the patient experience. In this article, the authors present their preferred approach to diagnosis and initial treatment, while highlighting common pitfalls and some useful simple strategies for communicating the diagnosis.
PARKINSON'S DISEASE is a common, progressive neurodegenerative disorder, the prevalence of which rises with advancing age, with an estimated population prevalence of $0.85 \%$ in Australia. ${ }^{1}$ As the population ages and average life expectancy increases, the number of Australians living with Parkinson's disease is expected to increase over the coming decades. The general practitioner (GP) has a vital role in timely diagnosis, effective communication, referral and treatment, which can have a lasting impact on the patient experience.

\section{Diagnosing Parkinson's disease}

While the patient with a classical presentation of Parkinson's disease, such as unilateral rest tremor and shuffling gait, can be easily recognised, there are patients in whom the diagnosis can be more elusive. Patients without tremor may have their symptoms erroneously attributed to another neurological condition, or the generalised slowness and balance disturbance may mistakenly be attributed simply to ageing. Patients may present with somatic complaints, such as constipation, or orthopaedic problems including shoulder pain. ${ }^{2}$ Such patients are often first referred to a rheumatologist, orthopaedic surgeon or psychiatrist before the diagnosis of Parkinson's disease is suspected. Conversely, there are a number of 'red flags' that should alert the clinician to the possibility of a diagnosis other than Parkinson's disease (Box 1), warranting earlier neurological review or investigation. Parkinson's disease mimics, which are summarised in Table 1 , should be considered when patients present with one or more of these red flags. While ancillary tests such as neuroimaging can be helpful to lend support to the diagnosis and to exclude mimics, Parkinson's disease remains a clinical diagnosis, and a careful history and examination are critical in recognising the diagnosis.

\section{Parkinson's disease is a whole-body disorder, not just a motor disease}

Parkinson's disease is now recognised as a whole-body disorder, affecting the gastrointestinal system in particular, but also the autonomic nervous system, sleep and mood in addition to giving rise to motor symptoms. Many of the non-motor symptoms develop before the onset of motor symptoms, giving rise to the concept of prodromal Parkinson's disease. Knowledge of these symptoms may facilitate early diagnosis and aid effective treatment by recognising the impact of non-motor manifestations on disability and quality of life.

\section{Motor symptoms}

The cardinal manifestations of Parkinson's disease are bradykinesia, or slowness of 


\section{Box 1. Red flags indicating that a diagnosis other than Parkinson's disease should be suspected}

- Objective limb weakness (note Parkinson's disease patients often report subjective weakness, with power found to be normal when formally tested)

- Rapidly progressive neurological deterioration over weeks to months

- Early, recurrent falls

- Absence of typical non-motor features (constipation, anosmia, rapid eye movement sleep disorder, daytime urinary urgency)

- Current use of dopamine-blocking medications, such as prochlorperazine, metoclopramide or antipsychotic medications

- Severe orthostatic hypotension ( $>30 \mathrm{mmHg}$ )

- Early cognitive impairment

- Urinary incontinence

- Lack of levodopa responsiveness

Table 1. Parkinson's disease mimics and when to consider

\begin{tabular}{ll}
\hline $\begin{array}{l}\text { Parkinson's disease } \\
\text { mimic }\end{array}$ & Characteristics \\
\hline $\begin{array}{l}\text { Essential or } \\
\text { dystonic tremor }\end{array}$ & $\begin{array}{l}\text { Tremor is absent at rest but becomes apparent with posture } \\
\text { (eg with the arms outstretched) or when using the hands. } \\
\text { Handwriting is usually large and shaky, rather than the micrographia } \\
\text { typical of Parkinson's disease. Tremor can also affect the head, neck } \\
\text { and voice. Other motor and non-motor signs of Parkinson's disease } \\
\text { are typically absent. }\end{array}$ \\
\hline
\end{tabular}

Medication-induced Recent or prolonged exposure to dopamine-blocking medications

Parkinsonism (prochlorperazine, metoclopramide, antipsychotic medications).

Typically bilateral and symmetrical Parkinsonism; however, can mimic typical Parkinson's disease.

Normal pressure hydrocephalus

Prominent gait freezing with broad-based gait and ventriculomegaly on neuroimaging (computed tomography or magnetic resonance imaging), particularly if combined with urinary incontinence and cognitive impairment.

\section{Progressive} supranuclear palsy

Early falls (particularly backwards) with postural instability.

Early cognitive slowing or personality change.

Poor levodopa response.

Multiple system

atrophy

Early, severe autonomic signs, especially urinary incontinence, erectile dysfunction and orthostatic hypotension.

Cerebellar signs (broad-based gait, limb incoordination, action tremor).

Poor levodopa response.

Dementia with

Fluctuating cognition.

Lewy bodies

Early, well-formed visual hallucinations.

Dementia occurring concurrently with, or preceding, motor symptoms.

movement, combined with rest tremor and/or rigidity. Limb bradykinesia is required for the diagnosis, but particularly movement that reduces in amplitude with repetition, termed motor decrement.
As the patient walks into the consulting room, you may notice they are shuffling or not swinging one arm. During the examination, it is important to look for reduced amplitude in movement or momentary arrest of movement with repetitive finger and toe tapping. Other manifestations of bradykinesia that may be evident from the examination include a mask-like facies due to reduced spontaneous facial movement, reduced blink rate, a hypophonic (soft) voice and micrographia.

The rest tremor of Parkinson's disease is typically slow $(4-6 \mathrm{~Hz})$, most commonly in the distal upper limb and asymmetrical, although lower limb onset can occur. In the upper limbs, this is best assessed by having the patient rest their hands in the prone or semi-prone position on their lap, or by having the patient hang their hands by their sides. ${ }^{3}$ Asking the patient to perform a simple mental task, such as saying the months of the year backwards, will often bring out a rest tremor. The patient with rest tremor almost certainly has a Parkinsonian syndrome, not essential tremor.

Early gait changes are characterised by asymmetrically reduced armswing, but over time stride length becomes shorter with a reduced step height, giving the gait its characteristic shuffle. The ability to pivot and turn may be lost, and patients turn en bloc, using multiple steps to turn. Falls are a late feature, and occurrence within the first year of diagnosis should arouse suspicion of a Parkinson's disease mimic (Table 1).

\section{Non-motor symptoms}

While Parkinson's disease is primarily defined by its motor symptoms, a broad but characteristic set of non-motor symptoms is present in most patients. It is often these non-motor symptoms that bother patients most and have been shown to have a greater negative impact on patient-perceived quality of life than the motor manifestations. ${ }^{4}$ These symptoms may sometimes appear vague but are often the first reason for presentation to the GP, and failure to appreciate their significance can lead to delays in diagnosis and heightened patient anxiety.

Rapid eye movement sleep behaviour disorder (RBD) occurs in approximately one-third of patients with Parkinson's disease and can precede the onset of motor symptoms by more than a decade. ${ }^{5}$ 
RBD occurs because of a failure to paralyse muscles during dreaming that occurs in normal sleep, resulting in dream enactment behaviour that can include vocalisations, thrashing movements, falls out of bed and unintentional injury to a partner. Patients are usually unaware of RBD as they are asleep, and this symptom needs to be elicited from a partner. Up to $80 \%$ of those with isolated RBD severe enough to be referred to a sleep clinic will eventually develop Parkinson's disease. Prodromal Parkinson's disease may be further suggested by the presence of constipation, which can predate motor manifestations by more than 10 years, ${ }^{5}$ and hyposmia. Constipation and hyposmia are present at some stage in the disease course in $70 \%$ and $90 \%$ of patients, respectively. ${ }^{6}$

Neuropsychiatric manifestations are common. The incidence of anxiety and depression is twice that of the general population and can appear at any stage of the illness, including as a prodromal symptom. Mood disturbance is the major determinant of health-related quality of life in patients with Parkinson's disease. With later disease, apathy, visual hallucinations and psychosis become increasingly prevalent. Visual hallucinations will eventually develop in up to $40 \%$ of patients with Parkinson's disease and are usually non-threatening, although more disturbing psychotic symptoms become more common as the disease progresses. Dementia eventually occurs in $40 \%$ of patients with Parkinson's disease, and it is related to both age and disease duration. ${ }^{6}$ Pain is an underrecognised feature and can be musculoskeletal, dystonic, neuropathic or central in origin, in some cases responding to dopaminergic therapy. ${ }^{7}$ Other non-motor features include excessive daytime somnolence, fatigue, insomnia and fragmented sleep, restless legs syndrome, sexual dysfunction, dysphagia and autonomic dysfunction (including orthostatic dizziness, bladder dysfunction, erectile dysfunction and hyperhidrosis).

\section{Delivering the diagnosis}

The GP's role is critical in a patient's experience with Parkinson's disease, and communication is an essential part of this, particularly in the early days where uncertainty regarding the diagnosis may exist. Patient satisfaction with the explanation of Parkinson's disease at the time of diagnosis has been shown to have a lasting impact on health-related quality of life, even many years after the diagnosis has been made. ${ }^{8}$ Perceived diagnostic delay and the involvement of more than one additional healthcare provider have been associated with patient dissatisfaction in the diagnostic process. ${ }^{9}$ In contrast, patient satisfaction has been strongly associated with more sensitive delivery of the diagnosis, the helpfulness of the information provided and whether an opportunity to ask questions was provided. ${ }^{10}$ Allowing patients the space for questions and expressions of anxiety can be crucial in their overall experience of the diagnostic process.

Patient preconceptions regarding Parkinson's disease vary greatly and are influenced by many factors. ${ }^{11}$ Most people have encountered someone with Parkinson's disease in their circle of acquaintance; others may have the experience of caring for a relative with Parkinson's disease. It is this picture of Parkinson's disease that patients may carry with them into the consultation room. Having recognised features (or researched their symptoms online), it is important to be cognisant of the fact that many patients will be worried about a diagnosis of Parkinson's disease, a concern not always actually voiced. If possible, it is preferable to deliver a diagnosis of Parkinson's disease with a spouse or support person present. After disclosing the clinical suspicion, it may be useful to broach the patient's understanding and feelings regarding Parkinson's disease. What do they know about Parkinson's disease? Knowledge gaps are a leading factor in patient and clinician disconnect. ${ }^{11,12}$ Significant misinformation, as well as information, exists in the ether about Parkinson's disease. It is worthwhile highlighting that Parkinson's disease is a clinical diagnosis based on history and examination findings, and that while some tests should be performed, they often play a supportive role.
No single approach will suit every patient or situation. Breaking bad news of a neurodegenerative condition requires skill and sensitivity while still communicating accurate information clearly. While it may not be appropriate to overburden an individual with complex information at the first consultation, a clear explanation of Parkinson's disease, its manifestations and the route to diagnosis should be communicated. Explanation of non-motor symptoms is a crucial component not to overlook; as mentioned previously, these are often the most disabling symptoms from the patient's perspective. Patients will undoubtedly worry about how their life may change and how Parkinson's disease will affect their work, activities of daily living, leisure activities and social life. It is easy to see how an initial interaction is thus crucial to forming an individual's outlook on the disease. While the final decision ultimately lies with the patient, these authors advise an overall positive approach with encouragement, once time has been taken to consider and accept the diagnosis, to not be defined by the diagnosis but instead to treat Parkinson's disease like any chronic illness, one to manage and live alongside while still focusing on maintaining a normal life. Specific advice may need to be adapted in later stages, but it is important to reinforce that Parkinson's disease progresses slowly and that there are effective symptomatic treatments available. Reassurance should be given that you as their GP, in addition to non-GP specialist services, will be available to help manage issues and symptoms should they arise. Patients should be afforded sufficient time to voice their questions (Box 2), and a follow-up consultation at a short interval may be useful to address further concerns and serve to provide reassurance that support is available. Some patients may find regional and local patient advocacy groups, such as Parkinson's Australia, a useful resource for support and education. However, there is danger in 'information overload', including overexposure to knowledge about late-stage complications, which may result in excessive fear and anxiety that can become the main source of disability. Online information should not replace the 
vital communication and support received from GPs, specialist neurologists and nurse services. Above all, shared decision making and patient-centred care should be among the core tenets communicated to patients during the diagnostic process. ${ }^{13,14}$

\section{Initial treatment}

Arriving at, and communicating, a diagnosis of Parkinson's disease may be complex. As a chronic, progressive disease, the majority of, if not all, patients should be referred to a specialist neurology service. How soon a patient is referred will depend on many factors including patient preference, GP resources and local availability of specialist services. If available, early referral to a specialist Parkinson's disease nurse can be invaluable. However, referral to specialist services does not necessitate a delay in treatment initiation. The decision to start treatment in patients again relies on patient preference, the severity of both non-motor and motor disability and related impairment in quality of life.
Other factors that influence timing of therapy initiation include occupation, age and presence of comorbidities. ${ }^{15}$ During the consultation, it is therefore necessary to identify which symptoms affect the patient's quality of life and function. In some patients, motor disability may be related to the presence of hand tremor, whereas for others overall slowness may be the chief complaint. While some manifestations may not seem pronounced to an external examiner, even perceived mild symptoms in a dominant hand, for example, can produce significant functional impairment and should thus influence the therapeutic plan. Dopaminergic therapy has the potential to effectively target these deficits through amelioration of bradykinesia, rigidity and tremor.

Levodopa, in combination with carbidopa or benserazide (both dopa decarboxylase enzyme inhibitors, inhibiting the peripheral breakdown of levodopa before it reaches the central nervous system), is the most effective therapy to control motor symptoms in patients with Parkinson's disease. There

\section{Box 2. Frequently asked questions and suggested explanations}

\section{Do I need treatment?}

Treatment should be initiated if there is any disability, either social or physical. Effective treatments are available for the motor symptoms of Parkinson's disease (tremor, stiffness, slowness) but also to help problems such as constipation, light-headedness, insomnia, anxiety and depression. Delaying treatment is of no benefit. The medications used are well established and typically well tolerated.

\section{What are my treatment options?}

Treatment should be focused on improving quality of life and may consist of a combination of medications and non-pharmacological therapies including exercise and physical therapy.

\section{What is my prognosis?}

Parkinson's disease is a condition that progresses slowly, with major change over as long as 5-10-year intervals. There is significant variability from person to person, and patients should be counselled that they cannot use the experience of others they know with Parkinson's disease as a guide.

\section{Will my driving and work be affected?}

Many factors may influence driving ability including vision/eyesight, cognition/attention, sleepiness, medications and disease severity. Most people with Parkinson's disease remain active and safe drivers; however, the licensing authorities may require annual medical clearance.

\section{Is Parkinson's hereditary? Are my family at risk?}

Most cases of Parkinson's disease are not hereditary. Approximately $10 \%$ of those with Parkinson's disease have a family member also affected. However, usually there are many factors involved rather than a single gene. Having one close family member with Parkinson's disease increases the risk of developing Parkinson's disease to approximately $2 \%$.

Note: A useful resource for patients is www.parkinsons.org.au

is no rationale to delaying levodopa therapy if there is anything more than mild disability, especially in older patients. Withholding levodopa therapy in the false hope that it will delay the later onset of motor fluctuations, such as peak dose dyskinesia, is inappropriate and deprives the patient of effective, early treatment. In patients with mild Parkinson's disease, monoamine oxidase-B (MAO-B) inhibitors are a good option because of their tolerability and once-daily administration. Dopamine agonists, either oral or patch formulation, are also effective for motor control in mild Parkinson's disease. However, patients should be advised regarding the potential risk of impulse control disorders from dopamine agonists, including compulsive gambling, eating, spending or hypersexuality. Furthermore, dopamine agonists may exacerbate excessive daytime somnolence and hallucinations. Thus, every consultation is an opportunity to probe for these potential side effects. Dopamine agonists should be commenced gradually (start low, go slow) because of the risk of nausea and dizziness. If symptoms are inadequately controlled with initial treatment with a dopamine agonist or MAOB-B inhibitor, there should be no hesitation to adding levodopa, often effective at a relatively low dose $(100 \mathrm{mg}$ three times per day). An overview of these treatments, and medications commonly prescribed for non-motor symptoms, are summarised in Tables 2 and 3.

Most patients with moderate Parkinson's disease symptoms have an excellent response to levodopa treatment, usually with initial doses of between $300 \mathrm{mg}$ and $600 \mathrm{mg}$ per day, divided into three doses through the day (morning, midday and night). Elderly patients may be intolerant to initial high doses because of nausea or dizziness; therefore, a slower titration is suggested. For example, prescribing a single dose of $50 \mathrm{mg} /$ day for 3-7 days, and gradually increasing the daily dose in $50 \mathrm{mg}$ increments at similar intervals, to an initial target dose of $100 \mathrm{mg}$ three times per day, is a safe approach. Following this scheme, doses can be gradually increased up 450-600 mg/day according to the patient's symptoms. Patients who do not respond to levodopa should 
Table 2. Commonly prescribed medications for Parkinson's disease and their side effects

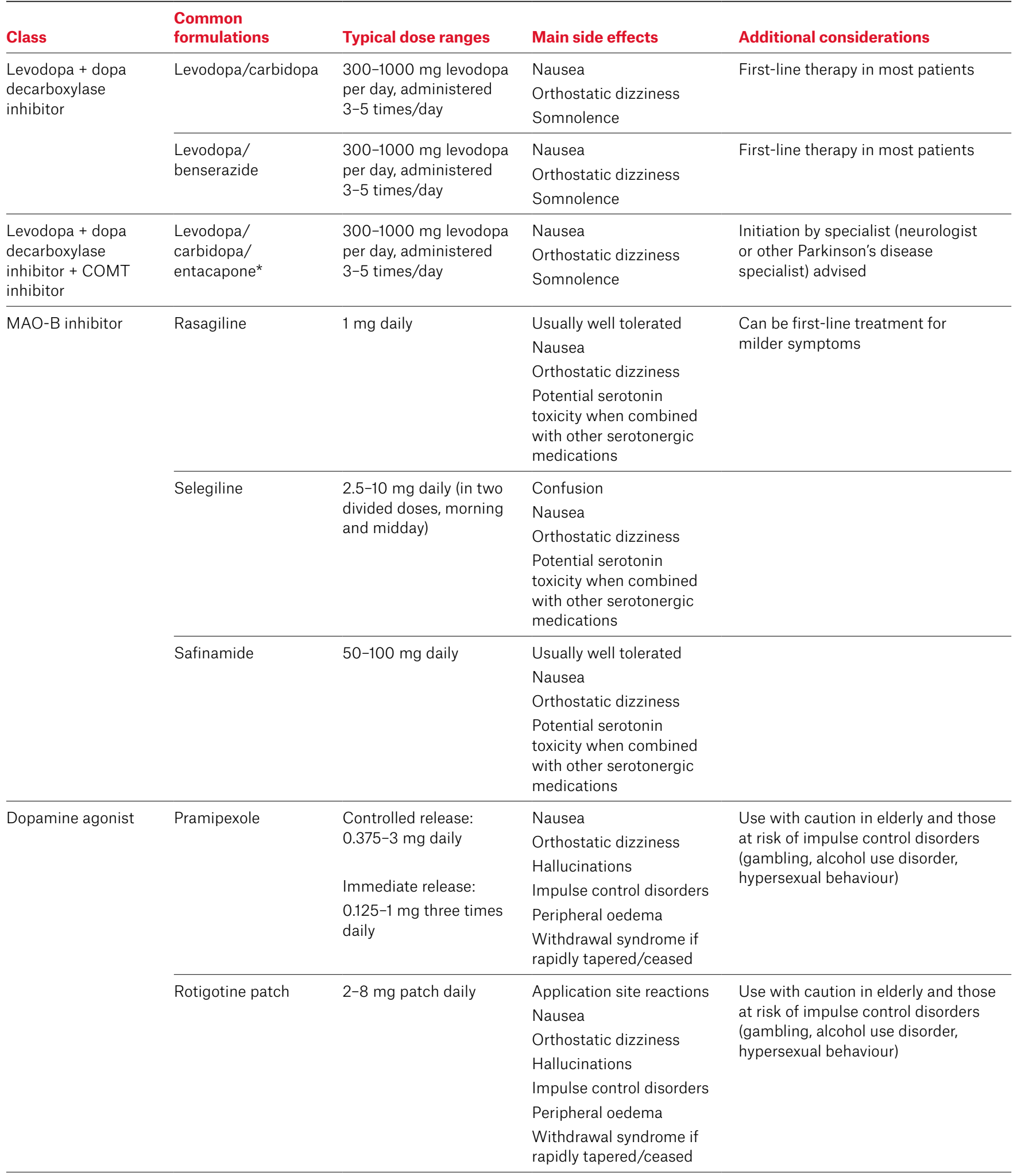


Table 2. Commonly prescribed medications for Parkinson's disease and their side effects (Cont'd)

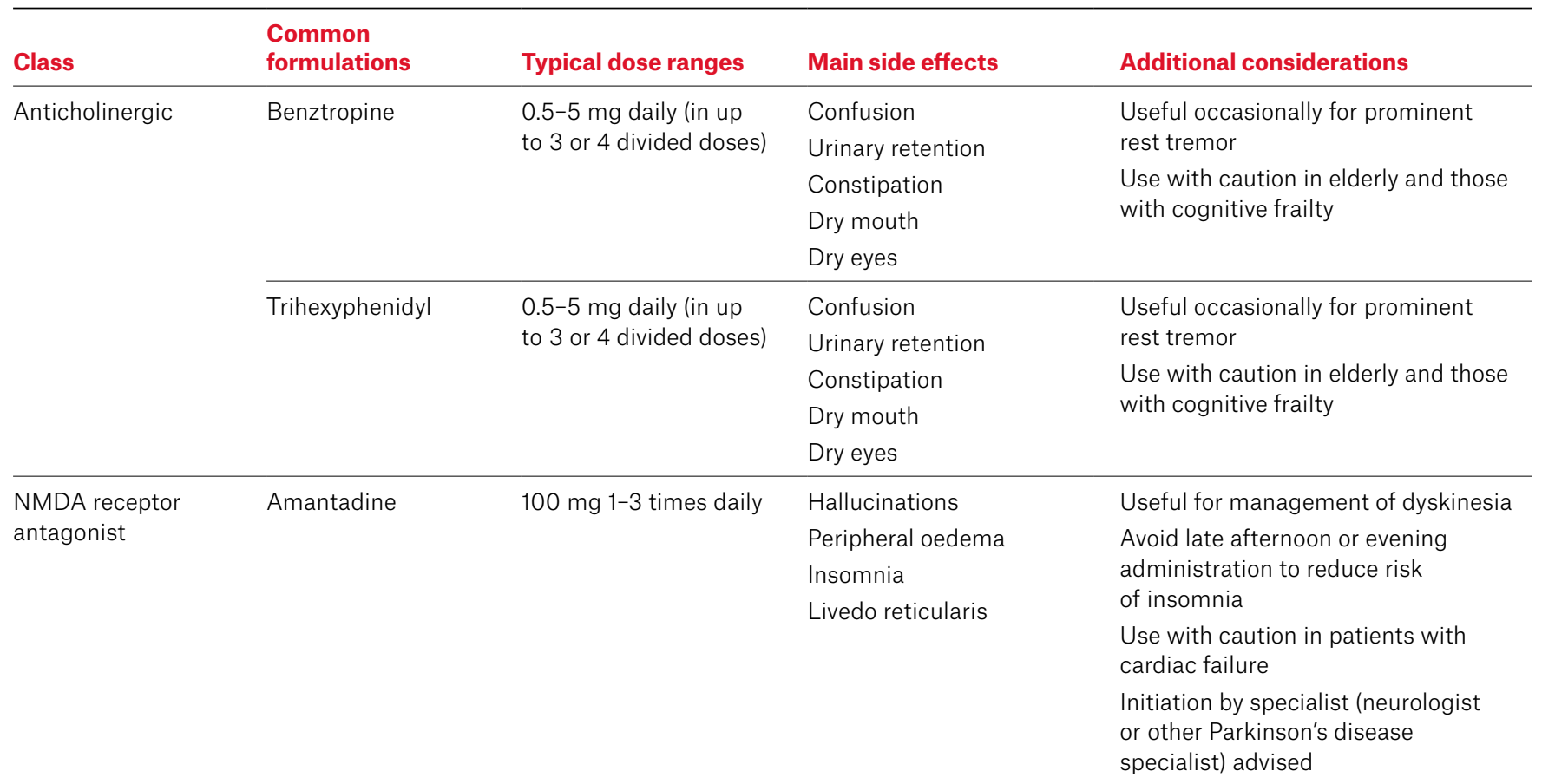

*Entacapone is a COMT inhibitor and works to prolong the action of levodopa. This can be particularly useful for patients whose Parkinson's disease symptoms reappear before their next medication dose is due.

COMT, catechol-O-methyltransferase; MAO-B, monoamine oxidase-B; NMDA, N-methyl-D-aspartate

be referred early to a neurologist for re-evaluation of the diagnosis. Many patients will have encountered common myths regarding levodopa therapy, such as levodopa therapy only being effective for five years, being toxic or promoting motor complications such as dyskinesia (medication-induced involuntary movements), leading to 'levodopa phobia'. It is worthwhile exploring whether patients harbour these misconceptions and reassuring them about the safety and efficacy of symptomatic treatment.

Exercise and physical therapies should be considered a core component of early treatment and management of Parkinson's disease. These authors' practical approach is to advise patients to endeavour to maintain the activities they enjoy, with a combination of aerobic, resistance, active balance and, if possible, high-intensity interval training. The positive effects of complex, multifaceted exercise programs, including dance and agility training, may extend beyond motor symptoms to cardiovascular status, cognitive, affective and sleep benefits, ${ }^{16}$ thus early referral to allied health services, such as physiotherapy and occupational therapy, may facilitate maintaining these skills and interests. Physiotherapy is of proven benefit in the short to medium term for patients with Parkinson's disease, ${ }^{17}$ particularly at early disease stages, and can target functional ability with the aims of minimising secondary complications of physical inactivity including carer dependence and social isolation.

Strategies employed should target specific underlying impairment, and areas of focus include gait, balance, transfers and upper limb function. ${ }^{18}$

While neurology support should be at hand, the GP is often the first port of call for patients with early symptoms or for individuals with an established Parkinson's disease diagnosis seeking treatment and advice. An effective initial approach should encompass prompt recognition of the core features of Parkinson's disease; clear explanation of the diagnosis and symptoms, allowing time for patient questions; and timely initiation of first-line therapy, complemented by effective, open communication between GP, patient and neurologist.

\footnotetext{
Authors

Sophie Waller BMed (Hons), Fellow, Movement Disorder Unit, Department of Neurology, Westmead Hospital, Westmead, NSW

Laura Williams MB, BCh, BAO, MD, Movement Disorder Unit, Department of Neurology, Westmead Hospital, Westmead, NSW

Hugo Morales-Briceño MD, Consultant Neurologist/ Senior Researcher, Movement Disorder Unit, Department of Neurology, Westmead Hospital, Westmead, NSW

Victor SC Fung FRACP, PhD, Director, Movement Disorder Unit, Department of Neurology, Westmead Hospital, Westmead, NSW

Competing interests: VSCF receives a salary from NSW Health; has received unrestricted research grants from the Michael J Fox Foundation, Abbvie and Merz; is on advisory boards and/or has received travel grants from Abbvie, Allergan, Ipsen, Merz, Praxis, Seqirus, Stada, Teva and UCB; and receives royalties from Health Press Ltd.

Provenance and peer review: Commissioned, externally peer reviewed.

Funding: None.

Correspondence to:

sophie.waller@health.nsw.gov.au
} 
Table 3. Commonly prescribed interventions for non-motor symptoms

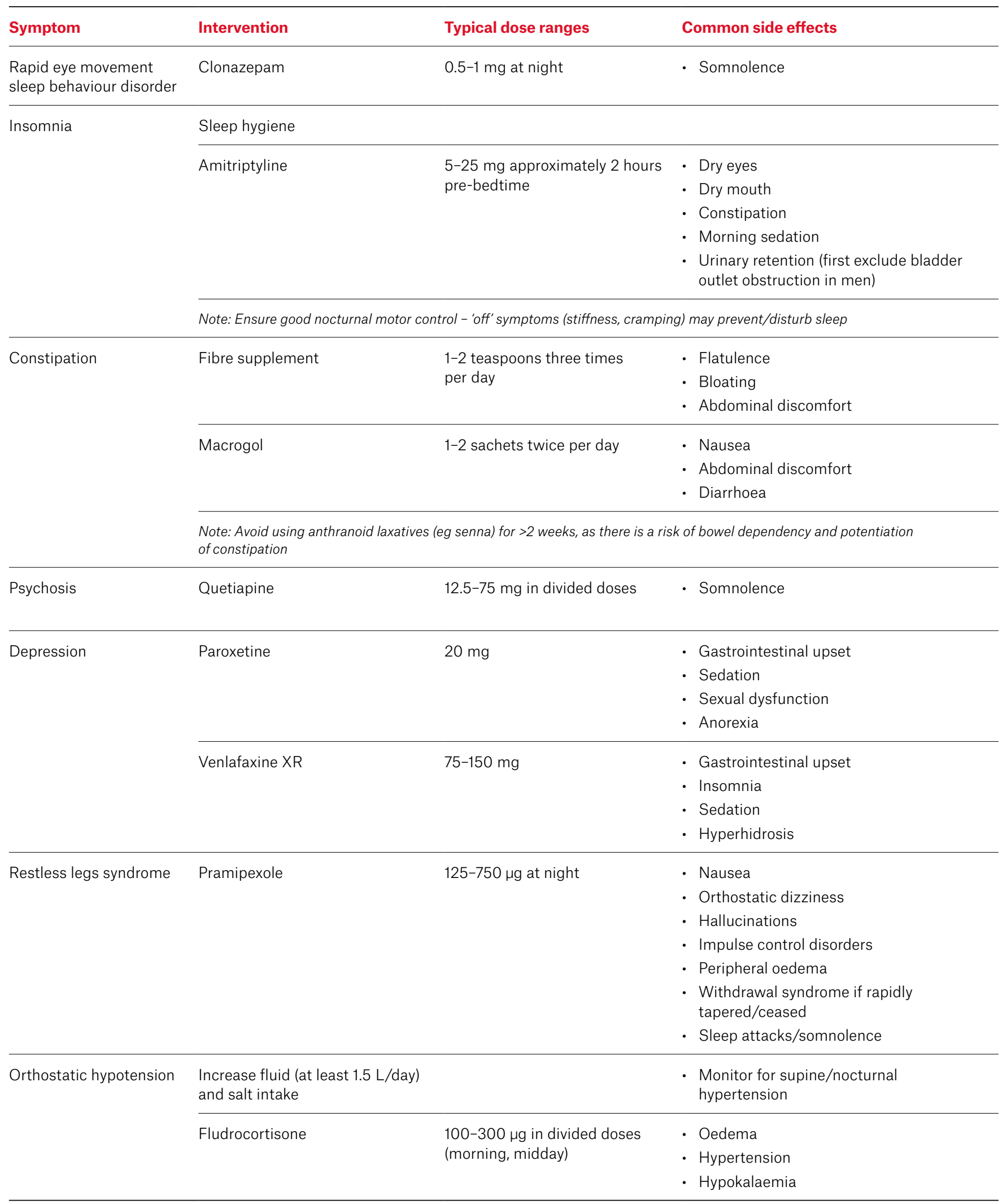




\section{References}

1. Ayton D, Ayton S, Barker AL, Bush Al, Warren N. Parkinson's disease prevalence and the association with rurality and agricultural determinants. Parkinsonism Relat Disord 2019;61:198-02. doi: 10.1016/j.parkreldis.2018.10.026.

2. Williams DR, Lees AJ. How do patients with parkinsonism present? A clinicopathological study. Intern Med J 2009;39(1):7-12. doi: 10.1111/j.14455994.2008.01635.x

3. Wilken M, Bruno V, Rossi M, Ameghino L, Deuschl G, Merello M. Sensitivity and specificity of different hand positions to assess upper limb rest tremor. Mov Disord 2019;34(4):575-79. doi: 10.1002/mds.27648.

4. Santos-García D, de la Fuente-Fernández R. Impact of non-motor symptoms on health-related and perceived quality of life in Parkinson's disease. J Neurol Sci 2013;332(1-2):136-40. doi: 10.1016/j. jns.2013.07.005.

5. Abbott RD, Petrovitch $H$, White LR, et al. Frequency of bowel movements and the future risk of Parkinson's disease. Neurology 2001;57(3):456-62. doi: 10.1212/wnl.57.3.456.

6. Chaudhuri KR, Healy DG, Schapira AH; National Institute for Clinical Excellence. Non-motor symptoms of Parkinson's disease: Diagnosis and management. Lancet Neurol 2006;5(3):235-45. doi: 10.1016/S1474-4422(06)70373-8.

7. $\mathrm{Ha} A D$, Jankovic J. Pain in Parkinson's disease. Mov Disord 2012;27(4):485-91. doi: 10.1002/ mds.23959.

8. Global Parkinson's Disease Survey (GPDS) Steering Committee. Factors impacting on quality of life in Parkinson's disease: Results from an international survey. Mov Disord 2002;17(1):60-67. doi: $10.1002 / m d s .10010$.

9. Plouvier AOA, Olde Hartman TC, de Bont OA, et al. The diagnostic pathway of Parkinson's disease: A cross-sectional survey study of factors influencing patient dissatisfaction. BMC Fam Pract 2017;18(1):83. doi: 10.1186/s12875-0170652-y.

10. Schrag A, Modi S, Hotham S, et al. Patient experiences of receiving a diagnosis of Parkinson's disease. J Neurol 2018;265(5):1151-57. doi: 10.1007/ s00415-018-8817-8.

11. Bhidayasiri R, Panyakaew $P$, Trenkwalder $C$, et al. Delivering patient-centered care in Parkinson's disease: Challenges and consensus from an international panel. Parkinsonism Relat Disord 2020;72:82-87. doi: 10.1016/j. parkreldis.2020.02.013.

12. Tan $A H$, Tan CT, Marras $C$, et al. Knowledge of Parkinson's disease in a multiethnic urban Asian setting. J Parkinsons Dis 2015;5(4):865-79. doi: 10.3233/JPD-150594.

13. Schrag A, Khan K, Hotham S, Merritt R, Rascol O, Graham L. Experience of care for Parkinson's disease in European countries: A survey by the European Parkinson's Disease Association. Eur J Neurol 2018;25(12):1410-e120. doi: 10.1111/ ene.13738.

14. National Institute for Health and Care Excellence. Parkinson's disease in adults [NG71]. London, UK: NICE, 2017.

15. de Bie RMA, Clarke CE, Espay AJ, Fox SH, Lang AE. Initiation of pharmacological therapy in Parkinson's disease: When, why, and how. Lancet Neurol 2020;19(5):452-61. doi: 10.1016/S14744422(20)30036-3.

16. Speelman $A D$, van de Warrenburg $B P$, van Nimwegen M, Petzinger GM, Munneke M, Bloem BR. How might physical activity benefit patients with Parkinson disease? Nat Rev Neurol 2011;7(9):528-34. doi: 10.1038/nrneurol.2011.107.
17. Tomlinson CL, Patel S, Meek C, et al. Physiotherapy intervention in Parkinson's disease: Systematic review and meta-analysis. BMJ 2012;345:e5004. doi: 10.1136/bmj.e5004.

18. van der Kolk NM, King LA. Effects of exercise on mobility in people with Parkinson's disease. Mov Disord 2013;28(11):1587-96. doi: 10.1002/ mds.25658. 